\title{
EISCAT observations of pump-enhanced plasma temperature and optical emission excitation rate as a function of power flux
}

\author{
C. J. Bryers, ${ }^{1}$ M. J. Kosch, ${ }^{1,4}$ A. Senior, ${ }^{1}$ M. T. Rietveld, ${ }^{2}$ and T. K. Yeoman ${ }^{3}$ \\ Received 2 May 2012; revised 21 June 2012; accepted 17 July 2012; published 6 September 2012.
}

[1] We analyze optical emissions and enhanced electron temperatures induced by high power HF radio waves as a function of power flux using the EISCAT heater with a range of effective radiated powers. The UHF radar was used to measure the electron temperatures and densities. The Digital All Sky Imager was used to record the $630.0 \mathrm{~nm}$ optical emission intensities. We quantify the HF flux loss due to self-absorption in the D-region (typically 3-11 dB) and refraction in the F-region to determine the flux which reaches the upper-hybrid resonance height. We find a quasi-linear relationship between the HF flux and both the temperature enhancement and the optical emission excitation rate with a threshold at $\sim 37.5 \mu \mathrm{Wm}^{-2}$. On average $\sim 70 \%$ of the HF flux at the upper-hybrid resonance height goes in to heating the electrons for fluxes above the threshold compared to $\sim 40 \%$ for fluxes below the threshold.

Citation: Bryers, C. J., M. J. Kosch, A. Senior, M. T. Rietveld, and T. K. Yeoman (2012), EISCAT observations of pumpenhanced plasma temperature and optical emission excitation rate as a function of power flux, J. Geophys. Res., 117, A09301, doi:10.1029/2012JA017897.

\section{Introduction}

[2] Artificially induced aurora by high frequency (HF) radio waves has been studied since the early 1970's [e.g., Biondi et al., 1970; Haslett and Megill, 1974]. The high power HF radio waves interact with the plasma via electrostatic resonances, accelerating electrons to high energies. The electrons then collide with the neutrals, predominantly $\mathrm{O}$ and $\mathrm{N}_{2}$ in the F-region of the ionosphere, causing photons of auroral wavelengths to be emitted. Electrons with energies above $1.96 \mathrm{eV}$ excite the $\mathrm{O}\left({ }^{1} \mathrm{D}\right)$ state which, upon relaxation, produces the well-known $630.0 \mathrm{~nm}$ emission. Artificial aurorae at the EISCAT, Tromsø, HF heater facility were first observed in February 1999 [Brändström et al., 1999; Kosch et al., 2000]. These optical signatures were associated with high electron temperature enhancements (up to $3500 \mathrm{~K}$ ) as measured by the UHF incoherent scatter radar, [e.g., Rietveld et al., 2003], co-located with the heater.

[3] Although the mechanisms to induce the artificial aurora are still not fully understood, the primary mechanism is thought to be upper-hybrid resonance (UHR) [Leyser et al., 2000]. This is supported by observations of artificial

\footnotetext{
${ }^{1}$ Department of Physics, Lancaster University, Lancaster, UK.

${ }^{2}$ EISCAT Scientific Association, Ramfjordmoen, Norway.

${ }^{3}$ Department of Physics and Astronomy, University of Leicester, Leicester, UK.

${ }^{4}$ School of Physics, University of KwaZulu-Natal, Durban, South Africa.

Corresponding author: C. J. Bryers, Department of Physics, Lancaster University, Lancaster LA1 4YB, UK. (c.bryers@lancaster.ac.uk)

C2012. American Geophysical Union. All Rights Reserved. 0148-0227/12/2012JA017897
}

aurora, which minimize when pumping on a gyroharmonic frequency where the UHR turbulence also minimizes [Kosch et al., 2000]. The UHR height is the altitude in the ionosphere at which the heater frequency $f_{\mathrm{h}}$, is equal to the upperhybrid (UH) frequency:

$$
f_{h}=f_{\mathrm{UH}}=\left(f_{p}^{2}+f_{e}^{2}\right)^{1 / 2}
$$

where $f_{e}$ is the electron gyrofrequency and $f_{p}$ is the plasma frequency. At this height, electrostatic waves perpendicular to the magnetic field line develop from the pump electromagnetic wave [Robinson, 1989]. By heating the plasma, electrostatic waves produce field-aligned striations; i.e. small scale, (1-10 $\mathrm{m}$ in size, across the geomagnetic field), regions of reduced electron density [Stocker et al., 1992; Robinson, 2002] within which the UH waves become trapped. Hence, pump-induced UH waves and striations are symbiotic. Coherent HF backscatter acts as a proxy to detect the presence of UH turbulence as the diagnostic radar wave undergoes Bragg scattering from the striations. Hence, the CUTLASS SuperDARN radar in Finland is able to detect UHR striations induced by the EISCAT heater [Bond et al., 1997].

\section{Modeling}

[4] We wish to quantitatively determine the relationship between the pump wave power flux and the induced artificial auroral intensity and electron temperature enhancements. To do this we consider the varying ionospheric conditions to determine the D-region absorption losses of the HF wave as well as the beam divergence due to refraction in the F-region. 


\subsection{Artificial Aurora Intensities}

[5] To accurately measure the auroral intensity enhancement, the images were background subtracted. Since observations are normally executed immediately after sunset, the background solar intensity decreases rapidly with time as the solar zenith angle changes. To accommodate for this change, two background images were used, one before and one after each pump-on period. During the relatively short pump-on cycle the decrease in the solar intensity was approximately linear so the background intensity could be calculated by interpolation separately for each of the data images.

[6] The intensity of the pixels inside the $-3 \mathrm{~dB}$ locus of the pump beam were summed up and the average pixel intensity taken. After sunset the UHR height increases as $f_{0} F 2$ decreases and so the altitude of the $O\left({ }^{1} D\right)$ emissions will vary also. This means that a fixed quantum of HF power flux results in a photon volume emission rate which depends on altitude. To correctly relate the HF-power to the production of $\mathrm{O}\left({ }^{1} \mathrm{D}\right)$ by using the intensity of the $630.0 \mathrm{~nm}$ emissions we have to take the quenching of $\mathrm{O}\left({ }^{1} \mathrm{D}\right)$ into account. Gustavsson et al. [2001] showed that the volume emission rate of the $\mathrm{O}\left({ }^{1} \mathrm{D}\right), I_{6300}(z)$, and the number density of excited oxygen, $n_{\mathrm{O} 1 \mathrm{D}}$, are related by:

$$
I_{6300}(z)=A_{6300} n_{\mathrm{O} 1 \mathrm{D}}(z)
$$

where $A_{6300}=5.15 \times 10^{-3} \mathrm{~s}^{-1}$ is the Einstein coefficient for the transition from $\mathrm{O}\left({ }^{1} \mathrm{D}\right)$ to the $\mathrm{O}\left({ }^{3} P_{2}\right)$ ground state and $z$ is altitude.

[7] The continuity equation for $\mathrm{n}_{\mathrm{O} 1 \mathrm{D}}$ includes expressions for the drift and diffusion of the species since the lifetime of the emission is long [Bernhardt et al., 2000]. We are able to neglect the diffusion and drift terms since we integrate the intensity over the full width of the pump beam and so any movement of emission source would remain inside this volume for typical short pump periods (2-4 min). The steady state continuity equation gives:

$$
n_{\mathrm{O} 1 \mathrm{D}}=\tau_{\mathrm{eff}} q(z)
$$

where the $\mathrm{O}\left({ }^{1} D\right)$ effective lifetime, $\tau_{\text {eff, is altitude depen- }}$ dent and typically 25-50 $\mathrm{s}$ in the F-region ionosphere [Gustavsson et al., 2001] and $\mathrm{q}$ is the $\mathrm{O}\left({ }^{1} D\right)$ excitation rate.

[8] This means that the relationship between the volume emission rate and the excitation rate becomes:

$$
\left.I_{6300}(z)\right)=A_{6300} \tau_{\text {eff }} q(z)
$$

Integration of $I_{6300}(z)$ over all altitudes was performed by Gustavsson et al. [2001] to give:

$$
q=\frac{I_{6300}(t o t)}{A_{6300} \tau_{\mathrm{av}}}
$$

where the altitude averaged lifetime, $\tau_{\mathrm{av}}$ was shown to be equal to the lifetime determined by way of fitting an exponential decay curve to the total enhanced intensity of the artificial aurora just after pump-off.

\subsection{Refraction Model}

[9] To calculate the dilution or concentration of pump wave power due to refraction we used a modified version of the Jones 3D ray-tracing program to calculate the raypath from the heater array on the ground to the UHR height using the electron density profile from the EISCAT UHF radar [Jones and Stephenson, 1975]. The divergence or convergence of the pump waves were calculated by tracing three rays close together in the heater beam volume, through the ionospheric layers and determining by how much the area between these three rays alters compared to that of only free space divergence. The calculation was performed by finding the HF power flux flowing through the area between the three rays at a low altitude $(20 \mathrm{~km})$ where the electron density is low enough not to cause any significant refraction:

$$
P_{1}=\left(\frac{E R P}{4 \pi R_{1}^{2}}\right) A_{1}
$$

where $P_{1}$ is the heater power passing through the area $A_{1}$ at the range, $R_{1} \mathrm{~km}$ and ERP is the heater effective radiated power. The power flux $S$ in $\mathrm{Wm}^{-2}$ at the UHR height, $R_{2}$, is given by:

$$
S=\frac{P_{1}}{A_{2}}
$$

where $P_{1}$ is the same power from $R_{1}$ passing through the larger area, $A_{2}$ at the UHR height. The area has changed from $A_{1}$ to $A_{2}$ because of refraction and free space expansion combined. The ratio of power flux change, $F$, due only to refraction at the UHR height is as follows:

$$
F=S /\left(E R P /\left(4 \pi R_{2}^{2}\right)\right)
$$

where the denominator is the power flux at the UHR height due to free space expansion only.

\subsection{D-Region Absorption Model}

[10] Despite the heater power remaining constant it was necessary to include modeling of the D-region absorption for each heater pulse since it is dependent upon plasma density. Senior et al. [2010] developed a D-region absorption model which also accounts for the self absorption of the HF heater beam which becomes significant at high heater powers due to the temperature enhancements increasing the electron-neutral collision frequency. The model allows for time dependent modeling but since the pump-on period is long compared to the heating and cooling of electrons, an equilibrium state is assumed. This equilibrium state means that the heater has been switched on a sufficiently long time that the increased electron temperature has reached a constant level and will remain at this temperature until the heater is switched off. The model requires the electron density measurements from the UHF radar power profiles, which are handled in $300 \mathrm{~m}$ wide altitude steps in the height range of 55-120 km.

[11] The model solves the time dependent electron energy equation for temperature [Senior et al., 2010, equation (9)] which incorporates several mechanisms to calculate the cooling rate of the thermal electrons by collisions with neutral molecules in the ionosphere. We use the cooling rate derived by Pavlov [1998a] for the excitation of vibrational and rotational states of $\mathrm{N}_{2}$ molecules which are a function of electron temperature. For the electron energy transfer rate for excitation of vibrational $\mathrm{O}_{2}$ states we use the equations of Jones et al. [2003]. Furthermore, we use the equations of Pavlov [1998b] for the cooling rate due to the excitation of the $\mathrm{O}_{2}$ rotational states to complete the loss function. 
[12] The complex refractive index calculated is the Appleton-Hartree equation for parallel propagation to the magnetic field. It makes use of the Schunk and Nagy [1978] electron-neutral collision frequency equation [Schunk and Nagy, 1978, equation 42]. The electron density profile used for the D-region absorption model comes from $60 \mathrm{~s}$ integrated UHF radar data averaged before and after the pump on period. This is to ensure that pump-induced artifacts do not adversely affect the profiles since there is an apparent electron density reduction due to D-region temperature enhancement.

\subsection{Photon Energies}

[13] Once the aforementioned processes have all been considered the heater power flux reaching the UHR height is known. From here it is possible to determine the energy lost to heating the electrons and creating the artificial aurora, to extract an efficiency estimate of the heating mechanism. The column emission rate for $630.0 \mathrm{~nm}$ is given by Gustavsson and Eliasson [2008]:

$$
I_{6300}=10^{-6} A_{6300} \int_{0}^{\infty} \tau_{\text {eff }}\left[I_{5577}(z)+n_{O} \int_{0}^{\infty} \sigma(E) \delta I(E, z) d E\right] d z
$$

where $\delta I(E, z)$ is the enhanced differential electron flux as a function of altitude, $z$, and energy, $E$, and $\sigma$ is the electron impact excitation cross section. Gustavsson and Eliasson [2008] used optical multiwavelength $(630.0,557.7,844.6$ $427.8 \mathrm{~nm}$ ) data to determine the enhanced electron flux and find the non-thermal component of the electron energy spectrum for high energies. Determination of the thermal plasma parameters from the raw EISCAT radar data is performed in GUISDAP; a standard software package which assumes a Maxwellian distribution of electron energies [Lehtinen and Huuskonen, 1996]. This means that the measured temperature is biased to the low energy electrons, of which there are many orders of magnitude more than for high energy electrons.

[14] We can estimate the electron energy loss rate to the excited oxygen by using the column excitation rate and emission intensity. The electrons are accelerated by the pump wave which then excite the dominant $\mathrm{O}\left({ }^{1} D\right)$ state which can lose energy either by photon production or quenching. We can estimate the energy in the photons using $E=h f$ and the photon energy flux, $H$, in $\mathrm{Wm}^{-2}$ by $H=\phi E$ where $h$ is the Planck constant and $\phi$ is the number of photons emitted per second per $\mathrm{m}^{2}$ calculated from the intensity in rayleighs for all wavelengths measured by Gustavsson et al. [2005]. Using the definition of the rayleigh [Hunten et al., 1956], gives $H=2.6 \times 10^{-7} \mathrm{Wm}^{-2}$ where the emissions from wavelengths other than $630.0 \mathrm{~nm}$ have a negligible effect on the photon energy flux.

[15] This however does not consider the energy lost via quenching, which goes unobserved. The column excitation rate $q_{\mathrm{col}}=\frac{I_{3300}}{A_{6330} \tau_{\mathrm{av}}}$ multiplied by the $630.0 \mathrm{~nm}$ excitation threshold energy of $1.96 \mathrm{eV}$ gives the electron energy loss to the $\mathrm{O}\left({ }^{1} D\right)$ excited state in $\mathrm{Wm}^{-2}$. For typical F-region lifetimes $(35 \mathrm{~s})$ the total electron energy loss to exciting $\mathrm{O}\left({ }^{1} D\right)$ is $\sim 7 H$.

\subsection{Heat Source}

[16] We can calculate the energy that is being passed into the electrons by the heating mechanism using the electron energy equation described by Schunk and Nagy [1978, equation 68], given by:

$$
\sin ^{2} I \frac{\partial}{\partial z}\left(K^{e} \frac{\partial T_{e}}{\partial z}\right)+\sum Q_{e}+\sum L_{e}=0
$$

where $I$ is the angle of inclination of the Earth's magnetic field. Since this angle is $77^{\circ}$ for EISCAT, the factor can be neglected here. The heat source, $Q_{e}$, is the sum of the HF heater beam heating and the solar photoionization heating and $T_{e}$ is the electron temperature. $L_{e}$ is the sum of the electron energy cooling rates. These were taken from Campbell et al. [2004] for the vibrational excitation of $\mathrm{N}_{2}$ and Pavlov [1998a] for the rotational excitation of $\mathrm{N}_{2}$. The vibrational and rotational excitations of $\mathrm{O}_{2}$ were taken from Jones et al. [2003] and Pavlov [1998b] respectively. Both the cooling due to the excitation of the $\mathrm{O}\left({ }^{1} D\right)$ and elastic collisions with ions were taken from Schunk and Nagy [1978, equations 34 and 37].

[17] $K^{e}$ is the electron thermal conductivity given by Banks [1966] [Schunk and Nagy, 1978, equation 59]. The expression derived by Banks [1966] was done so by using simple mean free path considerations and was shown to be accurate in the E and F regions. Equation (10) assumes the electron temperature of the heated electrons is independent of time. This means that it can only be applied once a thermal steady state has been reached and therefore only toward the end of the pump-on period.

[18] We follow the procedure of Senior et al. [2012] to solve equation (10) for the heater contribution of the heat source, $Q_{\mathrm{HF}}$. The altitude dependent heat source from the pump wave was approximated by a Gaussian form:

$$
Q_{H F}=\frac{1}{w \sqrt{2 \pi}} J \exp \left(\frac{-\left(z-z_{0}\right)^{2}}{2 w^{2}}\right)
$$

where $J, z_{0}$ and $w$ are constants to be determined. The values of $J, z_{0}$ and $w$ give the height-integrated heating in $\mathrm{Wm}^{-2}$, the height at which most of the pump wave energy is deposited and the half-width of the Gaussian heat source, respectively. Solar photoionization is of the same form and can be determined during a pump-off period when $Q_{H F}=0$.

[19] Equation (10) was solved for $T_{e}$ and the difference between the solution and the UHF radar electron temperature measurement was minimized by least squares fitting using the Levenberg-Marquardt algorithm, until a solution was arrived at. Constraints were applied to the boundary value problem so that at $120 \mathrm{~km}$ the ion temperature and the electron temperature were equal, which was confirmed by the UHF radar data, and at altitudes above $500 \mathrm{~km}$ the electron temperature was assumed to be constant.

[20] To obtain the efficiency for the pump wave transmission of energy to the heated electrons, the height-integrated heating, $J$ is compared to the heater flux at the UHR height.

$$
e=\frac{J}{S}
$$




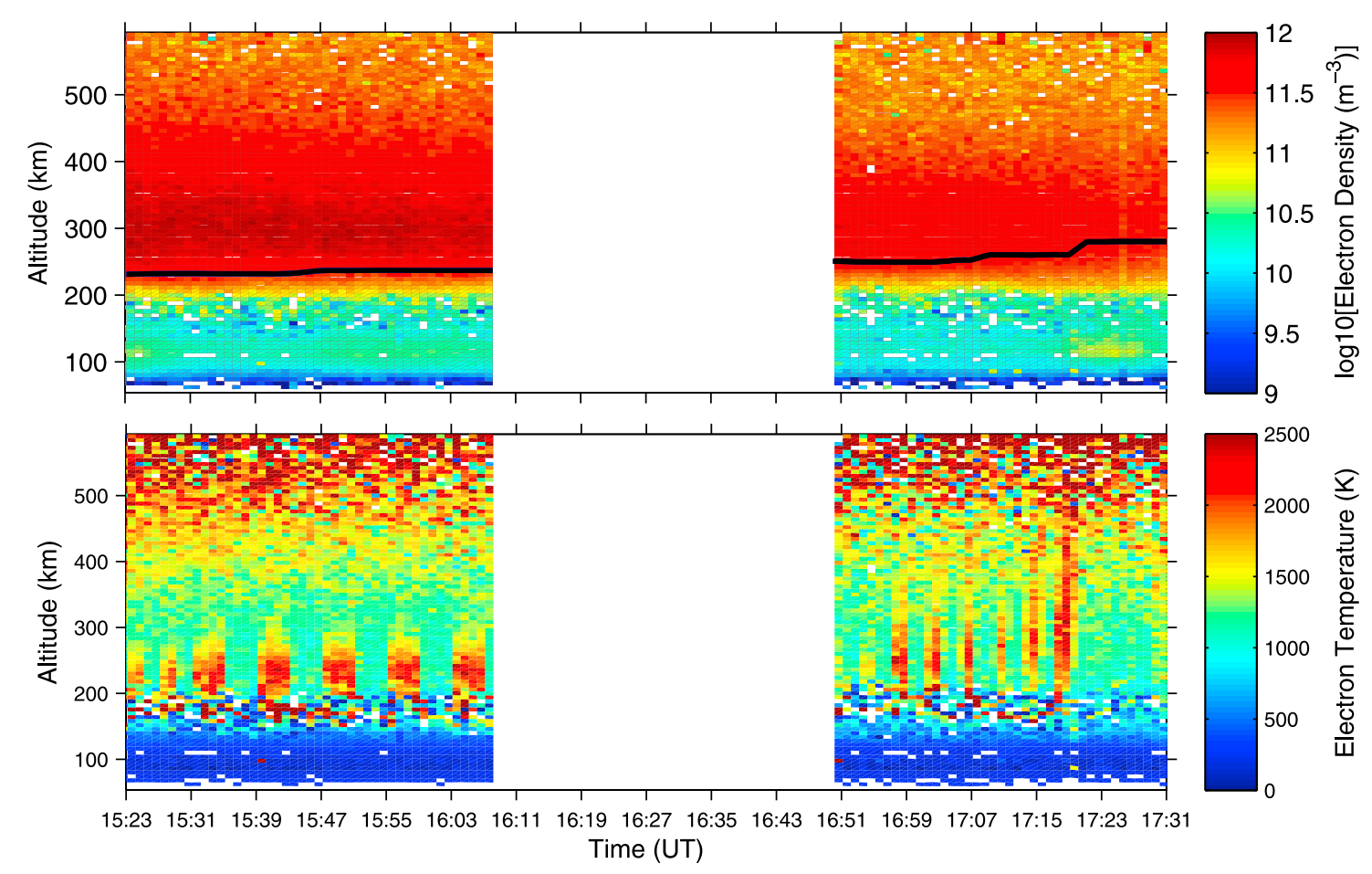

Figure 1. Electron (top) density and (bottom) temperature with altitude from $60 \mathrm{~s}$ integrated EISCAT UHF radar data. The black line in Figure 1 (top) shows the UHR height. The altitude resolution is $5.4 \mathrm{~km}$. The data gap was due to technical problems.

where $e$ is the efficiency and $S$ is the heater power flux reaching the UHR height.

\section{Experiment and Data}

[21] On 8 November 2001 from 15:15 to 17:30 UT, the HF heating facility [Rietveld et al., 1993] at EISCAT [Baron, 1984], near Tromsø, northern Norway $\left(69.58^{\circ} \mathrm{N}\right.$, $\left.19.21^{\circ} \mathrm{E}\right)$ was operated field-aligned $\left(13^{\circ} \mathrm{S}\right)$ in a power stepping mode. The heater frequency was fixed at $6.2 \mathrm{MHz}$ with O-mode polarization and a beam width of $7^{\circ}$. The heater was pulsed initially with 2 minute on, 2 minute off cycles, then 4 minute on, 4 minute off cycles after 15:31 UT at $100 \%$ power, switching to 2 minute on 2 minute off cycles with power stepping after 16:10 UT. This power stepping program comprised halving the heater power for each subsequent cycle from $100 \%$ (565 MW ERP) to $12.5 \%$ and then repeating the routine every $16 \mathrm{~min}$.

[22] The calculation of the ERP here assumes that the ground below the heater is perfectly reflective. An imperfect reflective surface would mean that some of the heater energy is absorbed in the ground and the ERP would be less. This consequently would alter the D-region absorption since it is dependent upon power. As a first approximation the ground absorption is constant for all heater powers and as a consequence of this, the ERP's presented here would be scaled by a constant factor. All previous studies at EISCAT assume the ground perfectly reflective so that comparisons with past results can be done easily. The reflectivity of the ground is dependent upon the materials it is composed of and the weather conditions on the day, such as snow cover. Measuring the day-to-day reflectivity would be difficult to do practically. Senior et al. [2011] estimated the true ERP's could be $75 \%$ of the currently used values.

[23] The EISCAT UHF radar was fixed in the field aligned pointing direction to obtain the electron temperature and electron density measurements. The radar code used was tau2 enabling measurements from 50 to $600 \mathrm{~km}$ in altitude with an unambiguous range resolution of $5.4 \mathrm{~km}$ and temporal resolution of $5 \mathrm{~s}$.

[24] The DASI optical system [Kosch et al., 1998] was imaging vertically $50 \mathrm{~km}$ away near Skibotn $\left(69.35^{\circ} \mathrm{N}\right.$, $\left.20.36^{\circ} \mathrm{E}\right)$. The camera had a $50^{\circ}$ field of view and a $630.0 \mathrm{~nm}$ filter fitted with an integration time of $10 \mathrm{~s}$.

[25] The Co-operative UK Twin-Located Auroral Sounding System (CUTLASS) HF radar at Hankasalmi, Finland $\left(62.32^{\circ} \mathrm{N}, 26.61^{\circ} 90 \mathrm{E}\right)$, part of the SuperDARN network [Greenwald et al., 1995; Chisham et al., 2007] was also operating at the time of the experiment to measure the coherent backscattered power from the pump-induced striations. Beam 5 was pointing over EISCAT with a range resolution of $45 \mathrm{~km}$ recording data every 2 minutes with an integration time of $7 \mathrm{~s}$. The heater was synchronized such that the CUTLASS measurements were made from 70-77 s after pump-on so that striations had enough time to grow to saturation, typically a few seconds [Grach et al., 2004]. The operating frequency was $12.4 \mathrm{MHz}$.

\section{Results and Discussion}

[26] Figure 1 shows the ionospheric electron density and temperature with altitude with a $60 \mathrm{~s}$ integration as measured by the UHF radar. The data gap was due to technical problems. The pump-on pulses can clearly be seen where the 
$15: 27: 40$

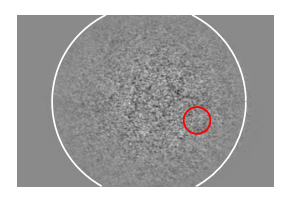

15:28:40

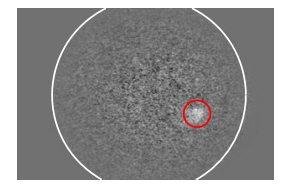

15:29:40

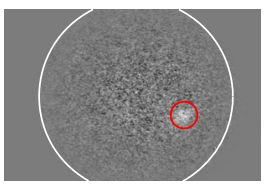

15:28:00

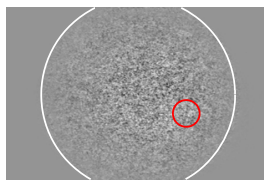

15:29:00

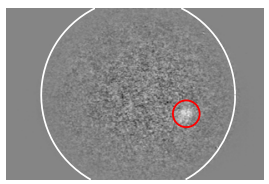

15:30:00

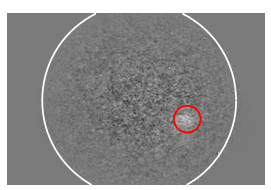

15:28:20

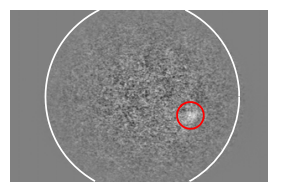

15:29:20

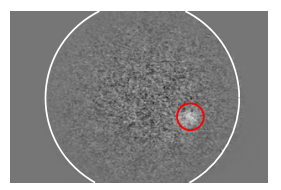

15:30:20

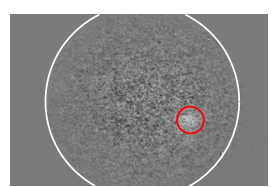

Figure 2. A sequence of ten second exposure images from DASI of the artificial aurora. The red circle shows the heater beam position at $240 \mathrm{~km}$ in altitude for field aligned pumping (13 ${ }^{\circ}$ South). The image wavelength is $630.0 \mathrm{~nm}$ with a $50^{\circ}$ field of view. The images have been background subtracted. North is at the top and East is on the left.

electron temperature is enhanced above background by up to $1200 \mathrm{~K}$. At around 17:20 UT the electron temperature enhancements cease as the ionospheric peak plasma frequency $\left(f_{o} F 2\right)$ falls below the heater frequency. When the ionosphere goes underdense the UHR condition normally ceases to be met soon thereafter (equation (1)) and consequently the electron temperature enhancement is significantly reduced and is due predominantly to radio wave ohmic heating of the plasma only [Gustavsson et al., 2010].

[27] Figure 2 shows a sequence of $630.0 \mathrm{~nm}$ images from the DASI imager with an integration time of $10 \mathrm{~s}$. The red circle shows the modeled heater beam $-3 \mathrm{~dB}$ locus at $240 \mathrm{~km}$ altitude for field-aligned pumping and the white circle shows the edge of the field of view. The sequence follows through time a typical pump-on pulse, which begins at 15:28 UT. The intensity of the artificial aurora increases up to a maximum until the steady state is reached at approximately 15:29 UT. The heater is turned off at 15:30 UT and the artificial aurora intensity decreases due to the height dependent decay time $(25-50 \mathrm{~s})$ of the $\mathrm{O}\left({ }^{1} \mathrm{D}\right)$ emission. The position of the artificial aurora in the sky changed due to refraction of the pump wave throughout the course of the experiment.

[28] Figure 3 shows an overview of the HF induced artificial aurora experiment. Figure 3 a shows the heater frequency in red (fixed at $6.2 \mathrm{MHz}$ ) and how $\mathrm{f}_{\mathrm{o}} \mathrm{F} 2$ changed in time (blue). After 17:20 UT $f_{\mathrm{h}}>f_{\mathrm{UH}}$ i.e. the ionosphere is underdense at $6.2 \mathrm{MHz}$, consistent with the electron temperature enhancements shown in Figure 1. Figure $3 \mathrm{~b}$ shows the $O\left({ }^{1} \mathrm{D}\right)$ background-subtracted optical intensity of the artificial aurora, averaged over the heater beam in rayleighs. Figure 3c shows the electron temperature at the UHR height. As the experiment proceeded the intensity of the artificial aurora and the electron temperature enhancement varied due to the stepping of the pump wave power and because of natural variations in the ionosphere which caused absorption and refraction of the HF pump wave. Note that the $O\left({ }^{1} D\right)$ emissions cease and electron temperature enhancements reduce significantly after 17:20 UT when the ionosphere went underdense. Figure $3 \mathrm{~d}$ shows the heater power flux at the UHR height, reduced by the D-region absorption and F-region refraction as well as beam divergence for free space propagation. The red line shows the threshold pump flux (discussed later). Figure 3e shows the heater on/off sequence at the different relative power levels it was transmitting. The red circles show the percentage of power reaching the UHR height (right hand side y-axis) which are in the range of 6$37 \%$ for the high and low powers respectively. Figure $3 f$ shows the UHR height (blue) calculated from equation (1) using 2-minute integrated UHF radar electron density profiles. The red circles show the height of the heat source, calculated in section 2.5 , which are within $\pm 10 \mathrm{~km}$ of the UHR heights for most cases and are discussed in more detail later. Toward the end of the experiment the UHR height becomes undefined when the ionosphere is underdense. Since the UHF radar beam is narrow $\left(0.6^{\circ}\right)$ compared to the heater beam $\left(7^{\circ}\right)$, some parts of the ionosphere may be either underdense or overdense within the heater beam. Therefore, the uncertainty between the UHR height and the height of the heat source becomes greater. The black circles show the altitude of the greatest temperature enhancement which tracks both the UHR height and the heat source height within $\pm 8 \mathrm{~km}$ for the majority of cases where the UHR height is well defined. Figure $3 \mathrm{~g}$ shows the self-absorption of the heater wave through the D-region, calculated in section 2.3, which varies as a function of heater power and is approximately $11 \mathrm{~dB}$ for high powers $(100 \%)$ and $3 \mathrm{~dB}$ for low powers (12.5\%). Figure $3 \mathrm{~h}$ shows the CUTLASS backscatter power from the 17 th range gate $(\sim 945 \mathrm{~km}$ range), on beam number 5 of the Hankasalmi radar which is directed over Tromsø. The data has two minute resolution and shows clearly that the backscattered power is significantly enhanced when the heater switches on. When the heater is switched off the backscattered power does not always fall back to zero since there is natural backscatter present and because the pump-induced striations have a long 


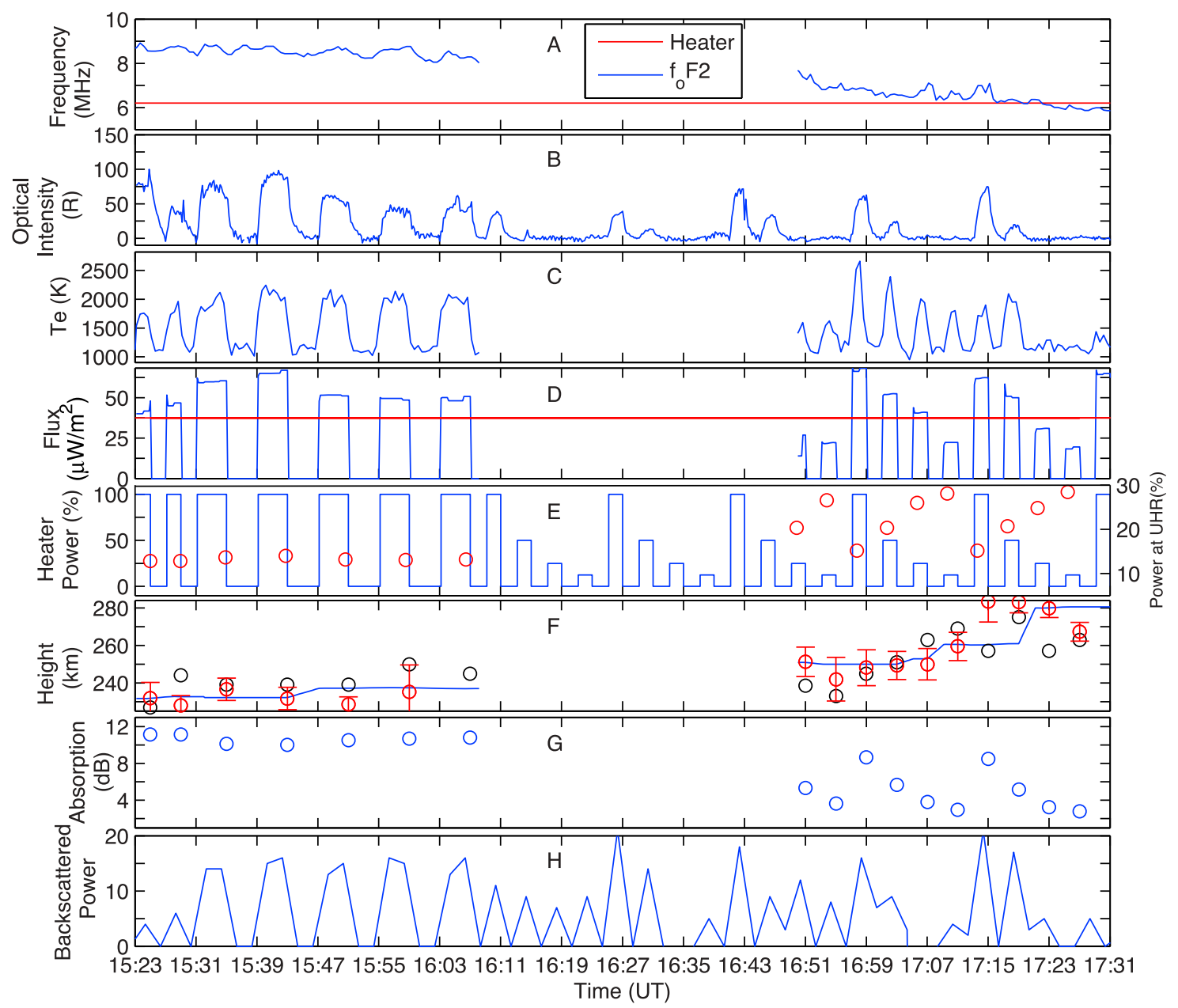

Figure 3. An overview of the ionospheric modification experiment on $08 / 11 / 2001$. (a) The heater frequency (red) and foF2 (blue). (b) The average optical intensity of the $630 \mathrm{~nm}$ emission above background. (c) The electron temperature at the UHR height. (d) The heater power flux at the UHR height adjusted for D-region absorption and refraction. The red line shows the threshold flux for UHR. (e) The heater on/off sequence and the transmitted power as a percentage. The red circles show the percentage of pump power reaching the UHR height (right hand side y-axis). (f) The UHR height (blue), the pump heat source height estimates (red) and the height of the highest temperature enhancement (black). (g) The modeled heater self-induced D-region absorption for a field-aligned $6.2 \mathrm{MHz}$ heater beam. (h) CUTLASS radar backscatter power for the 17th range gate over Tromsø.

decay time of the order of several minutes [Bond et al., 1997] at this time of day. The pump-induced backscattered power enhancements were not observed for the pump-on periods starting at 17:08 and 17:28 UT, indicating that UHR was not present. For other cases where the UHR flux threshold condition was not met some backscatter was observed. Unfortunately there was contamination of the data from natural backscatter from the south after 16:10 UT and natural backscatter to the north of the heated region during the whole experiment. This is probably the cause for the backscatter measurement even for cases where the pump flux is below the threshold.

[29] Figure 4 shows the flux ratio, $F$ from equation (8), for all pump-on periods in the experiment where electron density measurements were available (17 in total). The main inputs to the Jones 3D model, to determine the beam divergence, are the geographical location of the heater used to estimate the magnetic field strength, the angle of the beam, its polarization, power and frequency and the radar electron density profile. We used a median electron density profile averaged from 60 second resolution UHF radar profiles from pump-off periods before and after the current pump-on period as the input to the model. The electron densities were calibrated using the EISCAT Dynasonde. The UHF radar only provides us with a vertical electron density profile and so the refraction model does not consider any horizontal gradients which may have been present. However, plotting the refracted heater beam position onto the auroral images from the DASI camera cause the center of the beam to align well with the center of the artificial aurora indicating that horizontal gradient effects were negligible. The small oscillations in the raypaths, especially noticeable below $200 \mathrm{~km}$, are due to numerical errors in the ray tracing calculation.

[30] Performing the calculation described in section 2.2 at all ranges, $R$, we find beam focusing does occur at lower altitudes in some cases but the gain in power flux is small, at 


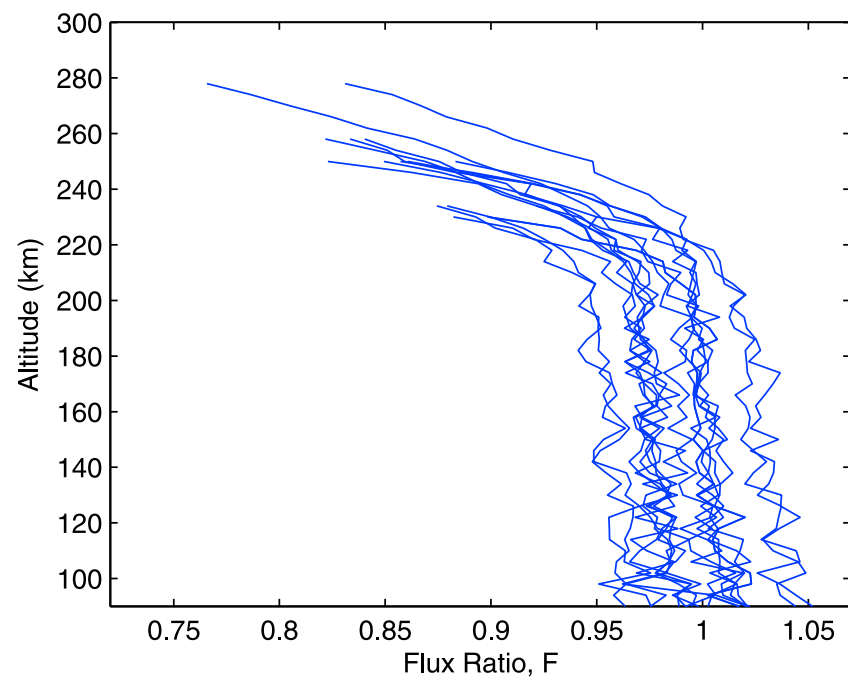

Figure 4. The relative change in pump power flux (flux ratio, F) due to HF refraction as a function of height. The raypaths stop at the UHR height.

around 5\%, which could be due to experimental uncertainties. The figure shows that the dominant F-region effect which decreases the heater power flux above $200 \mathrm{~km}$ altitude is the variation in the UHR height from the beginning of the experiment to the end. In this experiment $F$ varied from $90 \%$ to $75 \%$ as the UHR height increased from $\sim 230 \mathrm{~km}$ to $280 \mathrm{~km}$. For all pump-on periods we find the flux ratio is always less than 1 at the UHR height which indicates that beam defocusing was occurring.

\subsection{O( $\left.{ }^{1} D\right)$ Excitation Rate Relation to Pump Power Flux}

[31] We calculate the artificial auroral intensity, $I$ and average decay lifetime to determine the $\mathrm{O}\left({ }^{1} \mathrm{D}\right)$ excitation rate, q, for the 17 pump pulses of varying ERP. Figure 5 shows these results for the estimated heater power flux adjusted for D-region absorption, F-region refraction and free space beam-divergence. The colors of the data points indicate their pump power in percent as described in the figure legend. The uncertainty in the flux measurements were calculated by repeating the model calculation several times for varying electron densities within the range of the UHF radar uncertainties. The optical intensities were calculated by averaging the last 3 images for when the heater was on.

[32] For fluxes up to $30-35 \mu \mathrm{Wm}^{-2}$ the excitation rate is close to zero. Above a threshold of $37.5 \mu \mathrm{Wm}^{-2}$ the response to the increasing flux is a quasi-linear increase in excitation rate corresponding to a linear fit of:

$$
q=6.73 \times 10^{4} S-252.4 \times 10^{4}
$$

where $S$ is expressed in $\mu \mathrm{Wm}^{-2}$.

[33] Equation (13) was achieved by fitting two linear plots to the points both below and above the threshold. A HF threshold of $37.5 \mu \mathrm{Wm}^{-2}$ is estimated when $q=0$ and must therefore be exceeded to initiate the $\mathrm{O}\left({ }^{1} \mathrm{D}\right)$ emission. Due to little data being present close to the threshold, the value of

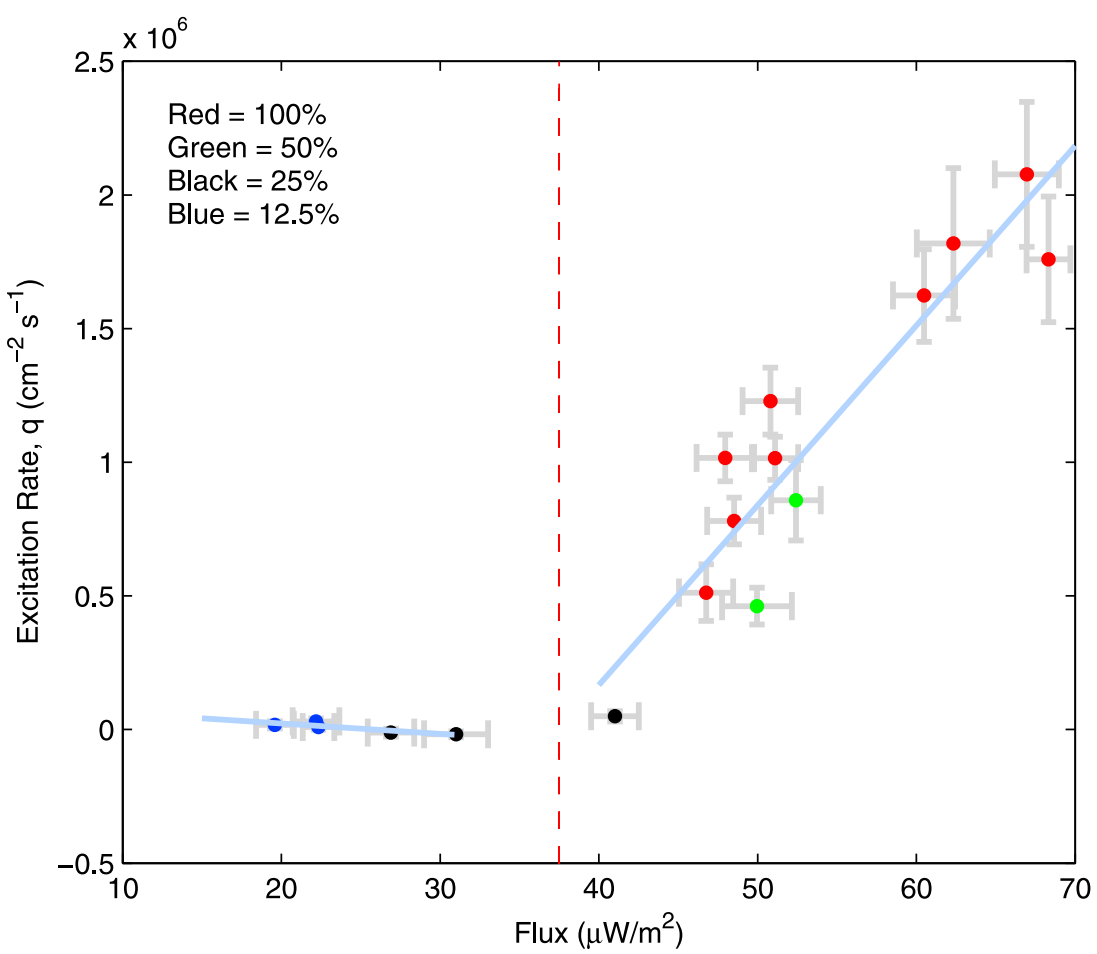

Figure 5. $O\left({ }^{1} D\right)$ excitation rate as a function of pump power flux at the UHR height. The data point colors correspond to the heater power level as shown by the figure legend, $100 \%=565$ MW ERP. Linear fits have been applied to points above and below the threshold. The dashed line at $37.5 \mu \mathrm{Wm}^{-2}$ shows the approximate threshold flux. 


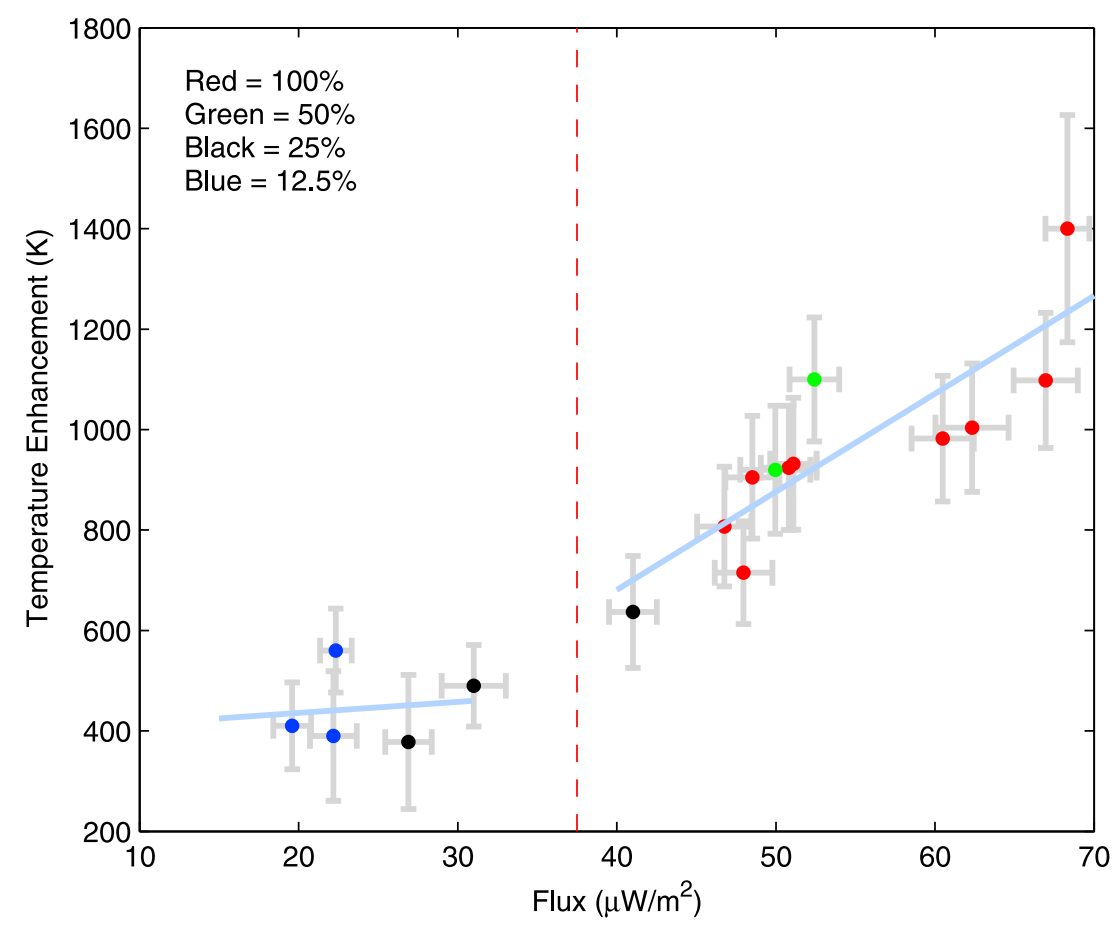

Figure 6. Electron temperature enhancement above background as a function of pump power flux at the UHR height. The data point colors correspond to the heater power level as shown by the figure legend, $100 \%=565$ MW ERP. Linear fits have been applied to points above and below the threshold. The dashed line at $37.5 \mu \mathrm{Wm}^{-2}$ shows the approximate threshold flux.

$37.5 \mu \mathrm{Wm}^{-2}$ given here is uncertain and we can only assume that the threshold lies somewhere in the range $32-$ $40 \mu \mathrm{Wm}^{-2}$. This result shows that the $\mathrm{O}\left({ }^{1} \mathrm{D}\right)$ emission can only be produced if the threshold flux is exceeded by the pump wave. The same is true for all other artificially stimulated auroral emissions since they all have a higher energy threshold than $O\left({ }^{1} \mathrm{D}\right)$.

[34] Since we have calculated the reduced heater power flux due to various loss mechanisms we can infer a "corrected' ERP that would be needed to produce this flux assuming no losses. The heater flux, $F_{R}$ at $R$ produced by the transmitter is:

$$
F_{R}=\frac{E R P}{4 \pi R^{2}}
$$

We set the threshold flux to be $37.5 \mu \mathrm{Wm}^{-2}$ taken from Figure 5. Using this value in equation (14) gives a threshold ERP of $\sim 29 \mathrm{MW}$. This is the minimum heater power needed to stimulate optical emissions in a lossless ionosphere.

[35] Assuming the ground below the heater was not perfectly reflective and the ERP was reduced by $25 \%$ [Senior et al., 2011], causes small ( $10-20 \%)$ reductions of the flux estimates with the greatest reductions occurring for the lower powers which are already below the threshold. This means that the trend and threshold presented here remains approximately the same to within the experimental uncertainty.

\subsection{Electron Temperature Enhancement Relation to Pump Power Flux}

[36] Figure 6 shows the electron temperature enhancement plotted for the same heater power fluxes as Figure 5. The electron temperature was measured at the UHR height which was close to being the highest temperature in the height profile below $300 \mathrm{~km}$ within the measurement uncertainties, shown in Figure 3f. The background temperature was found in the same way for data recorded two minutes later when the temperature had relaxed back to its unperturbed state after the heater had been switched off. We used $60 \mathrm{~s}$ integration times. All cases of pump on showed some temperature enhancement and the flux threshold at $37.5 \mu \mathrm{Wm}^{-2}$ is not as obvious as for the $O\left({ }^{1} D\right)$ excitation rate. The temperature enhancement for the lowest heater power fluxes below $37.5 \mu \mathrm{Wm}^{-2}$ cluster around $400 \mathrm{~K}$. Since the UHR threshold condition has not been met for fluxes below $37.5 \mu \mathrm{Wm}^{-2}$ this small temperature enhancement is due to electromagnetic wave ohmic heating only.

[37] Ohmic heating is due to a non-zero HF-conductivity and associated collisional damping of the HF wave. Löfas et al. [2009] studied the effect of X-mode heating in underdense conditions on the electron temperature enhancement. They looked at cases where no plasma resonance conditions were met where the sole plasma heating mechanism was electromagnetic ohmic heating and observed electron temperature enhancements of 300-400 K. Similarly Gustavsson et al. [2010] studied underdense O-mode heating and found temperature enhancements of order $500 \mathrm{~K}$. These are consistent with our low flux observations. If a quasi-constant electron temperature enhancement of $400 \mathrm{~K}$ is due to ohmic radio wave heating, then any additional temperature enhancements above the flux threshold are due to UHR. For this scenario, a flux threshold of $37.5 \mu \mathrm{Wm}^{-2}$ becomes obvious, which is consistent with the optical excitation rate threshold shown in Figure 5. 

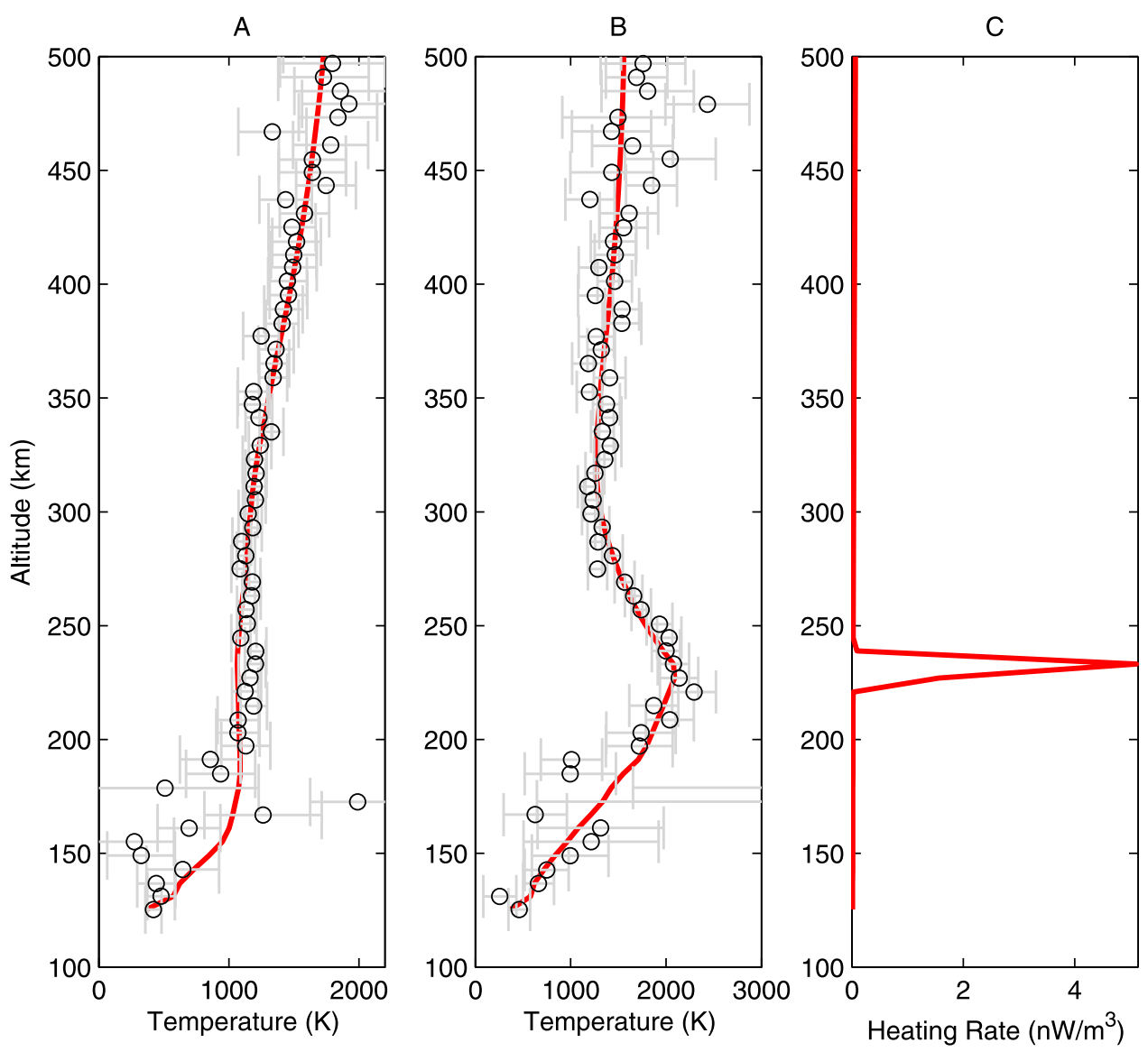

Figure 7. (a) The background electron temperature profile from EISCAT (black circles) and the modeled (red) electron temperature at 15:44 UT. (b) The heater enhanced electron temperature profile. (c) The Gaussian heat source at its predicted height.

[38] Referring back to Figure 3 we see that the enhancements in electron temperature for the $25 \%$ and $12.5 \%$ power heater pulses close to 17:07 UT are for the cases where the pump power flux is below or on the boundary of the threshold flux. We can also see from the CUTLASS radar data that there is an absence of backscattered power indicating that there were no striations present and we can assume that there was little or no heating via UHR. Here there is a noticeable increase in temperature but not in optical emissions, further indicating that the threshold of UHR has not been met and that UHR is responsible for the emissions. This suggests that the electron temperature enhancements in these cases must be mainly due to ohmic heating by the electric field of the pump wave itself. In these cases, the pump frequency is close to the critical frequency so that reflection occurs close to the F-region peak. It may be that the large scale height close to reflection leads to a larger distance over which the pump can interact with the plasma, increasing the efficiency of the heating.

[39] We have also applied a linear fit to the plot for the points above the threshold giving:

$$
\Delta T_{e}=19.53 S-100.53
$$

where $S$ is expressed in $\mu \mathrm{Wm}^{-2}$.

\subsection{Height Integrated Heating Relation to Pump Power Flux}

[40] Figure 7 shows a typical heat source profile using the method described in section 2.5. The black circles with gray error bars show the UHF measured electron temperature profile and the red line shows the modeled fit by solving equation (10). Figure 7 a shows the fitted model to the background, unheated electron temperature profile to estimate the heat source due to photoionization only. This was done for the last $60 \mathrm{~s}$ of each pump-off period to allow the ionosphere to cool back down to its unperturbed state. Figure $7 \mathrm{~b}$ shows the model fitted to a heated electron temperature profile at 15:44 UT where the heater power was $100 \%$ (565 MW). Figure 7c shows the Gaussian heat source due to the heater, $Q_{\mathrm{HF}}$.

[41] The heights of the fitted profiles can be seen in Figure $3 \mathrm{f}$ (red circles). For the majority of cases, the height at which the pump wave energy is absorbed falls within $\pm 5 \mathrm{~km}$ of the UHR height. The width of $Q_{\mathrm{HF}}$ is narrow, less than $\pm 5 \mathrm{~km}$ from the Gaussian peak for the majority of cases with a mean and standard deviation of 3.9 and $3.7 \mathrm{~km}$, respectively. This indicates that the energy is deposited by the HF pump wave in a narrow region which then propagates along the field line as the electrons are heated and accelerated, as seen in the electron temperature data in Figure 1. 


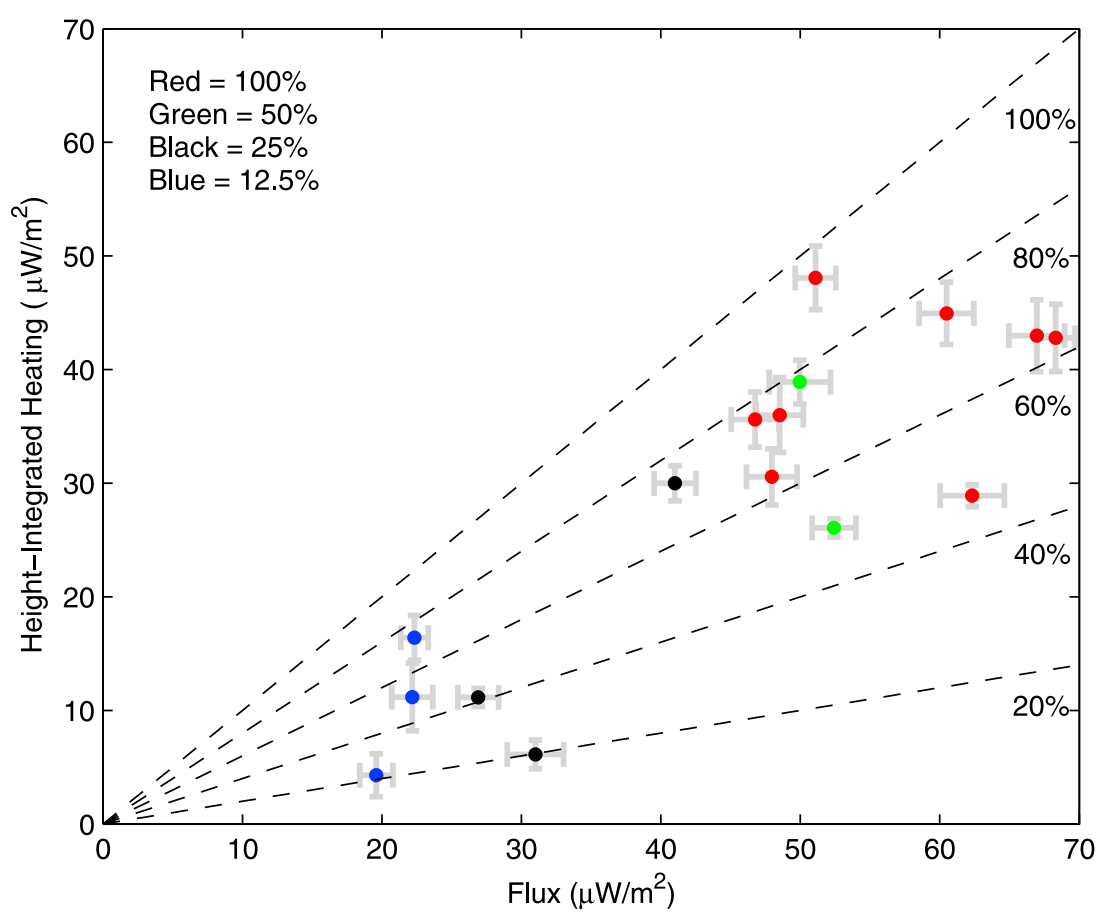

Figure 8. Modeled height-integrated heat source as a function of heater power flux at the UHR height. The black dashed lines represent efficiency levels and the data point colors correspond to the heater power level as shown by the figure legend, $100 \%=565 \mathrm{MW}$ ERP. The vertical dashed line at $37.5 \mu \mathrm{Wm}^{-2}$ shows the threshold flux.

Toward the end of the experiment the UHR altitude is illdefined when the ionosphere comes close to being underdense at the pump frequency of $6.2 \mathrm{MHz}$. Here it is found that the heating height is further away from the estimated UHR altitude and the width of $Q_{\mathrm{HF}}$ increases to $\pm 15 \mathrm{~km}$ at 17:19 UT probably because of the shallow gradient in plasma density near $\mathrm{f}_{\mathrm{o}} \mathrm{F} 2$.

[42] Figure 8 shows the height-integrated heating as a function of heater power flux at the UHR height. The dashed lines show the efficiency of the conversion of energy from the pump wave into the electrons as defined in equation (12). The efficiency is higher with increasing heater power flux which almost approaches $100 \%$ in one case. For fluxes below $37.5 \mu \mathrm{Wm}^{-2}$, corresponding to the lower heater ERP's, the efficiency averages at around $40 \%$ whereas the efficiency averages about $70 \%$ for the fluxes above the threshold. This is consistent with Senior et al. [2012]. This shows that the plasma heating mechanism is boosted from ohmic heating by electromagnetic waves to ohmic heating by electromagnetic and electrostatic UH waves and the efficiency increases. Stimulating UHR is therefore more efficient at heating the plasma than radio wave ohmic heating alone and necessary for accelerating the electrons above $1.96 \mathrm{eV}$ needed to produce the $630.0 \mathrm{~nm}$ emissions. This is also true for all other optical emissions which have greater excitation thresholds.

[43] The remaining energy, when pumping above the flux threshold ( $\sim 30 \%$ of the pump wave), must be therefore be reflected or scattered, or be transferred to the high energy electrons which are not detected by the UHF radar, some of which is emitted as auroral photons. Since the maximum intensity of the artificial aurora was $100 \mathrm{R}$ we can estimate the energy in the photons and the energy transferred to the $\mathrm{O}\left({ }^{1} \mathrm{D}\right)$ excited state using the method described in section 2.4. The observed $630.0 \mathrm{~nm}$ emission gives $H=3.2 \times$ $10^{-7} \mathrm{Wm}^{-2}$ which is 2 orders of magnitude less than the estimated HF flux where we have assumed other emission wavelength intensities as negligible. Our estimate shows that $\sim 1 \%$ of the pump flux at the UHR height is converted into photons. The total energy lost by the high energy electrons to the excited state of $\mathrm{O}\left({ }^{\mathrm{D}} \mathrm{D}\right)$ is $\sim 7 H$ (see section 2.4) and so approximately $7 \%$ of the total pump wave energy is lost to these processes.

[44] Frolov et al. [1997] found that the reflected pump wave intensity at SURA was around $-20 \mathrm{~dB}$, i.e. only $1 \%$ of the initial pump wave was reflected back. Stocker et al. [1993] measured the relative strength of a diagnostic wave during pump-off and pump-on periods. They measured a $10-12 \mathrm{~dB}$ loss due to anomalous absorption as the wave is scattered by the striations, i.e. $6-10 \%$ of the initial pump wave is reflected back. Similarly Fejer and Kopka [1981] found that $-10 \mathrm{~dB}$ of the pump wave was reflected back, indicating that the energy scattered by the diagnostic and the pump wave is approximately the same.

[45] Since, on average, $\sim 70 \%$ of the pump energy at the UHR height is transferred to the ionospheric plasma for cases with the pump flux above the threshold, we assume that some of the remaining energy available in Figure 8 (i.e. $\sim 30 \%)$ must be reflected back to the ground $(\sim 10 \%)$ which leaves $\sim 20 \%$ on average, which will be transferred to the suprathermal electrons not represented in the electron temperature enhancements shown in Figure 1. Of this 20\%, approximately a third is lost to the excitation of $\mathrm{O}\left({ }^{1} \mathrm{D}\right)$. 
[46] For our low power flux cases, where pump-induced striations were not present, the remaining pump energy was greater than for the high flux cases since only $\sim 40 \%$ of the wave is transferred to the electrons. This must mean that a greater amount of energy is reflected back down to the ground than at high flux cases.

\section{Summary and Conclusions}

[47] In this paper we have presented the empirical relationships between the pump wave power flux at the UHR height, enhanced electron temperature and $\mathrm{O}\left({ }^{1} \mathrm{D}\right)$ excitation rate. To perform such quantitative work, D-region selfabsorption and F-region refraction of the pump wave must be calculated. We have shown that a quasi-linear relationship between HF power flux and both $\mathrm{O}\left({ }^{1} \mathrm{D}\right)$ excitation rate and enhanced electron temperature exists with a threshold flux at $\sim 37.5 \mu \mathrm{Wm}^{-2}$. We have calculated the efficiency of the plasma heating process at $6.2 \mathrm{MHz}$, which averages about $40 / 70 \%$ for low/high HF power fluxes below/above the flux threshold. For electrons with a Maxwellian energy distribution we estimate that on average $\sim 40 \%$ of the pump energy goes into ohmic heating of the plasma and $\sim 30 \%$ is transferred to electrons via electrostatic UH waves; giving a larger heating efficiency for plasma resonance cases. We estimate that $\sim 20 \%$ of the pump wave energy is transferred to the suprathermal electrons, a third of which is lost to excitation of the $\mathrm{O}\left({ }^{1} \mathrm{D}\right)$ state where a total of $\sim 1 \%$ of the pump flux at the UHR altitude is converted into photons.

[48] Acknowledgments. EISCAT is an international scientific association supported by research organizations in China (CRIRP), Finland (SA), Japan (NIPR and STEL), Norway (NFR), Sweden (VR), and the United Kingdom (NERC).

[49] Robert Lysak thanks the reviewers for their assistance in evaluating this paper.

\section{References}

Banks, P. M. (1966), Charged particle temperatures and electron thermal conductivity in the upper atmosphere, Ann. Geophys., 22, 577-587.

Baron, M. (1984), The EISCAT facility, J. Atmos. Terr. Phys., 46, 469-472.

Bernhardt, P. A., M. Wong, J. D. Huba, B. G. Fejer, L. S. Wagner, J. A. Goldstein, C. A. Selcher, V. L. Frolov, and E. N. Sergeev (2000), Optical remote sensing of the thermosphere with HF pumped artificial airglow, J. Geophys. Res., 105, 10,657-10,671.

Biondi, A. A., D. P. Sipler, and R. D. Hake (1970), Optical $(\lambda-6300)$ detection of radio frequency heating of electrons in the F-region, J. Geophys. Res., 75, 6421-6424.

Bond, G. E., T. R. Robinson, P. Eglitis, D. M. Wright, A. J. Stocker, M. T. Rietveld, and T. B. Jones (1997), Spatial observations by the CUTLASS coherent scatter radar of ionospheric modification by high power radio waves, Ann. Geophys., 15, 1412-1421, doi:10.1007/ s00585-997-1412-4

Brändström, B. U. E., T. B. Leyser, A. Steen, M. T. Rietveld, B. Gustavsson, T. Aso, and M. Ejiri (1999), Unambiguous evidence of HF pumpenhanced airglow, Geophys. Res. Lett., 26, 3561-3564.

Campbell, L., M. J. Brunger, D. C. Cartwright, and P. J. O. Teubner (2004), Production of vibrationally excited N2 by electron impact, Planet. Space Sci., 52, 815-822.

Chisham, G., et al. (2007), A decade of the Super Dual Auroral Radar Network (SuperDARN): Scientific achievements, new techniques and future directions, Survey. Geophys., 28, 33-109.

Fejer, J. A., and H. Kopka (1981), The effect of plasma instabilities on the ionospherically reflected wave from a high-power transmitter, J. Geophys. Res., 86(A7), 5746-5750.

Frolov, V. L., L. M. Erukhimov, S. A. Metelev, and E. N. Sergeev (1997), Temporal behaviour of artificial small-scale ionospheric irregularities: Review of experimental results, J. Atmos. Sol. Terr. Phys., 59(18), 2317-2333, doi:10.1016/S1364-6826(96)00126-5.
Grach, S., E. Sergeev, A. Nasyrov, R. Gumerov, R. Shaimukhametov, I. Nasyrov, and G. Komrakov (2004), Simultaneous observations of the $557.7 \mathrm{~nm}$ airglow and stimulated electromagnetic emission during $\mathrm{HF}$ pumping of the ionosphere with diagnostic schedule: First results., $A d v$. Space Res., 34, 2422-2427.

Greenwald, R. A., et al. (1995), DARN/SUPERDARN: A global view of the dynamics of high-latitude convection, Space. Sci. Rev., 71, 761-796.

Gustavsson, B., and B. Eliasson (2008), HF radio wave acceleration of ionospheric electrons: Analysis of HF-induced optical enhancements, J. Geophys. Res., 113, A08319, doi:10.1029/2007JA012913.

Gustavsson, B., T. Sergienko, M. T. Rietveld, F. Honary, A. Steen, B. U. E. Brändström, T. B. Leyser, A. L. Aruliah, T. Aso, and M. Ejiri (2001), First tomographic estimate of volume distribution of enhanced airglow emission caused by HF pumping, J. Geophys. Res., 106, 29,105-29,123.

Gustavsson, B., et al. (2005), The electron energy distribution during HF pumping, a picture painted with all colors, Ann. Geophys., 23, 1747-1754.

Gustavsson, B., M. T. Rietveld, N. V. Ivchenko, and M. J. Kosch (2010), Rise and fall of electron temperatures: Ohmic heating of ionospheric electrons from underdense HF radio wave pumping, J. Geophys. Res., 115, A12332, doi:10.1029/2010JA015873.

Haslett, J. C., and L. R. Megill (1974), A model of the enhanced airglow excited by RF-radiation, Radio Sci., 9, 1005-1019.

Hunten, D. M., F. E. Roach, and J. W. Chamberlain (1956), A photometric unit for the airglow and aurora, J. Atmos. Terr. Phys., 8, 345-346.

Jones, D. B., L. Campbell, M. J. Bottema, and M. J. Brunger (2003), New electron energy transfer rates for vibrational excitation of $\mathrm{O}_{2}, \mathrm{New} \mathrm{J}$. Phys., 5(1), 114.1-114.11.

Jones, R. M., and J. J. Stephenson (1975), A versatile three-dimensional ray tracing computer program, Off. of Telecommun., Rep., 75-76, U.S. Govt. Print. Off., Washington, D. C.

Kosch, M. J., E. Nielsen, and T. Hagfors (1998), A new digital all-sky imager for optical auroral studies in conjunction with the Scandinavian twin auroral radar experiment, Rev. Sci. Instrum., 69, 578-584.

Kosch, M. J., T. Rietveld, T. Hagfors, and T. Leyser (2000), High-latitude HF-induced airglow displaced equatorward of the pump beam, Geophys. Res. Lett., 27, 2817-2820.

Lehtinen, M. S., and A. Huuskonen (1996), General incoherent scatter analysis and GUISDAP, Atmos. Terr. Phys., 58, 435-452.

Leyser, T. B., B. Gustavsson, B. U. E. Brändström, A. Steen, F. Honary, M. T. Rietveld, T. Aso, and M. Ejiri (2000), Simultaneous measurements of high-frequency pump-enhanced airglow and ionospheric temperatures at auroral latitudes, Polar Upper Atmos. Res., 14, 1-11.

Löfas, H., N. Ivchenko, B. Gustavsson, T. B. Leyser, and M. T. Rietveld (2009), F-region electron heating by X-mode radiowaves in underdense conditions,Ann. Geophys., 27, 2585-2592.

Pavlov, A. V. (1998a), New electron energy transfer rates for vibrational excitation of $\mathrm{N}_{2}$, Ann. Geophys., 16, 176-182.

Pavlov, A. V. (1998b), New electron energy transfer and cooling rates by excitation of $\mathrm{O}_{2}$, Ann. Geophys., 16, 1007-1013.

Rietveld, M. T., H. Kohl, H. Kopka, and P. Stubbe (1993), Introduction to ionospheric heating at Tromsø-I. Experimental overview, J. Atmos. Sol. Terr. Phys., 55, 577-599.

Rietveld, M. T., M. J. Kosch, N. F. Blagoveshchenskaya, V. A. Kornienko, T. B. Leyser, and T. K. Yeoman (2003), Ionospheric electron heating, optical emissions, and striations induced by powerful HF radio waves at high latitudes: Aspect angle dependence, J. Geophys. Res., 108(A4), 1141, doi:10.1029/2002JA009543.

Robinson, T. R. (1989), The heating of the high latitude ionosphere by high power radio waves, Phys. Rep., 179, 79-209.

Robinson, T. R. (2002), Effects of multiple scatter on the propagation and absorption of electromagnetic waves in a field-aligned-striated cold mageneto-plasma: Implications for ionospheric modification experiments, Ann. Geophys., 20, 41-55.

Schunk, R. W., and A. F. Nagy (1978), Electron temperatures in the F region of the ionosphere: Theory and observations, Rev. Geophys. 16(3), 355-399.

Senior, A., M. T. Rietveld, M. J. Kosch, and W. Singer (2010), Diagnosing radio plasma heating in the polar summer mesosphere using crossmodulation: Theory and observations, J. Geophys. Res., 115, A09318, doi:10.1029/2010JA015379.

Senior, A., M. T. Rietveld, F. Honary, W. Singer, and M. J. Kosch (2011), Measurements and modeling of cosmic noise absorption changes due to radio heating of the $\mathrm{D}$ region ionosphere, J. Geophys. Res., 116, A04310, doi:10.1029/2010JA016189.

Senior, A., M. T. Rietveld, T. K. Yeoman, and M. J. Kosch (2012), The dependence of F-region electron heating on HF radio pump power: Measurements at EISCAT Tromsø, J. Geophys. Res., 117, A04309, doi:10.1029/2011JA017267. 
Stocker, A. J., T. R. Robinson, and T. B. Jones (1992), Observations fo the effects of ionospheric heating on the amplitude of low-power diagnostic radio waves at Arecibo, J. Geophys. Res., 97, 6315-6322.
Stocker, A. J., F. Honary, T. R. Robinson, and T. B. Jones (1993), Anomalous absorption during artificial modification at harmonics of the electron gyrofrequency, J. Geophys. Res., 98, 13,627-13,634. 